\title{
Majorization for a Class of Analytic Functions Defined by $q$-Differentiation
}

\author{
K. A. Selvakumaran, ${ }^{1}$ Sunil Dutt Purohit, ${ }^{2}$ and Aydin Secer ${ }^{3}$ \\ ${ }^{1}$ Department of Mathematics, R.M.K College of Engineering and Technology, Puduvoyal, Tamil Nadu 601206, India \\ ${ }^{2}$ Department of Basic Sciences (Mathematics), College of Technology and Engineering, \\ M.P. University of Agriculture and Technology, Udaipur, Rajasthan 313001, India \\ ${ }^{3}$ Department of Mathematical Engineering, Yildiz Technical University, Davutpasa, 34210 Istanbul, Turkey
}

Correspondence should be addressed to Aydin Secer; asecer@yildiz.edu.tr

Received 24 May 2014; Accepted 1 July 2014; Published 10 July 2014

Academic Editor: Mustafa Bayram

Copyright (C) 2014 K. A. Selvakumaran et al. This is an open access article distributed under the Creative Commons Attribution License, which permits unrestricted use, distribution, and reproduction in any medium, provided the original work is properly cited.

We introduce a new class of multivalent analytic functions defined by using $q$-differentiation and fractional $q$-calculus operators. Further, we investigate majorization properties for functions belonging to this class. Also, we point out some new and known consequences of our main result.

\section{Introduction and Preliminaries}

Let $\mathscr{A}_{p}$ denote the class of functions $f(z)$ of the form

$$
f(z)=z^{p}+\sum_{n=p+1}^{\infty} a_{n} z^{n}, \quad(p \in \mathbb{N}=\{1,2,3, \ldots\}),
$$

which are analytic and $p$-valent in the open unit disk $\mathscr{U}=$ $\{z \in \mathbb{C}:|z|<1\}$. For analytic functions $f(z)$ and $g(z)$ in $\mathscr{U}$, we say that the function $f(z)$ is majorized by $g(z)$ in $\mathcal{U}$ (see [1]) and write

$$
f(z) \ll g(z) \quad(z \in \mathscr{U}),
$$

if there exists a Schwarz function $w(z)$, analytic in $\mathcal{U}$, such that

$$
|w(z)| \leq 1, \quad f(z)=w(z) g(z) \quad(z \in \mathscr{U}) .
$$

For the convenience of the reader, we now give some basic definitions and related details of $q$-calculus which are used in the sequel.

For any complex number $\alpha$ the $q$-shifted factorials are defined as

$$
(\alpha ; q)_{0}=1, \quad(\alpha ; q)_{n}=\prod_{k=0}^{n-1}\left(1-\alpha q^{k}\right), \quad n \in \mathbb{N},
$$

and in terms of the basic analogue of the gamma function

$$
\left(q^{\alpha} ; q\right)_{n}=\frac{\Gamma_{q}(\alpha+n)(1-q)^{n}}{\Gamma_{q}(\alpha)}, \quad(n>0),
$$

where the $q$-gamma function is defined by

$$
\Gamma_{q}(x)=\frac{(q, q)_{\infty}(1-q)^{1-x}}{\left(q^{x} ; q\right)_{\infty}}, \quad(0<q<1) .
$$

If $|q|<1$, the definition (4) remains meaningful for $n=\infty$ as a convergent infinite product

$$
(\alpha ; q)_{\infty}=\prod_{j=0}^{\infty}\left(1-\alpha q^{j}\right) .
$$

In view of the relation

$$
\lim _{q \rightarrow 1^{-}} \frac{\left(q^{\alpha} ; q\right)_{n}}{(1-q)^{n}}=(\alpha)_{n},
$$

we observe that the $q$-shifted factorial (4) reduces to the familiar Pochhammer symbol $(\alpha)_{n}$, where $(\alpha)_{n}=\alpha(\alpha+$ 1) $\cdots(\alpha+n-1)$. 
It may be noted that the $q$-Gauss hypergeometric function ${ }_{2} \Phi_{1}[-]$ (see Gasper and Rahman [2, p.3, eqn. (1.2.14)]) is defined by

$$
{ }_{2} \Phi_{1}[\alpha, \beta ; \gamma ; q, z]=\sum_{n=0}^{\infty} \frac{(\alpha ; q)_{n}(\beta ; q)_{n}}{(\gamma ; q)_{n}(q ; q)_{n}} z^{n}, \quad(|q|<1,|z|<1)
$$

and as a special case of the above series for $\gamma=\beta$, we have

$$
{ }_{1} \Phi_{0}[\alpha ;-; q, z]=\sum_{n=0}^{\infty} \frac{(\alpha ; q)_{n}}{(q ; q)_{n}} z^{n}, \quad(|q|<1,|z|<1) .
$$

Also, the $q$-derivative and $q$-integral of a function on a subset of $\mathbb{C}$ are, respectively, given by (see [2, pp. 19-22])

$$
\begin{gathered}
D_{q} f(z)=\frac{f(z)-f(z q)}{(1-q) z}, \quad(z \neq 0, q \neq 0), \\
\int_{0}^{z} f(t) d_{q} t=z(1-q) \sum_{k=0}^{\infty} q^{k} f\left(z q^{k}\right) .
\end{gathered}
$$

Therefore, the $q$-derivative of $f(z)=z^{n}$, where $n$ is a positive integer, is given by

$$
D_{q} z^{n}=\frac{z^{n}-(z q)^{n}}{(1-q) z}=[n]_{q} z^{n-1}
$$

where

$$
[n]_{q}=\frac{1-q^{n}}{1-q}=q^{n-1}+\cdots+1,
$$

and is called the $q$-analogue of $n$. As $q \rightarrow 1$, we have $[n]_{q}=$ $q^{n-1}+\cdots+1 \rightarrow 1+\cdots+1=n$. Here we list some relations satisfied by $[n]_{q}$ :

$$
\begin{gathered}
{[m+n]_{q}=[m]_{q}+q^{m}[n]_{q}=q^{n}[m]_{q}+[n]_{q},} \\
{[m-n]_{q}=q^{-n}[m]_{q}-q^{-n}[n]_{q},} \\
{[0]_{q}=0, \quad[1]_{q}=1 .}
\end{gathered}
$$

Recently, many authors have introduced new classes of analytic functions using $q$-calculus operators. For some recent investigations on the classes of analytic functions defined by using $q$-calculus operators and related topics, we refer the reader to [3-13] and the references cited therein. In the following, we define the fractional $q$-calculus operators of a complex-valued function $f(z)$, which were recently studied by Purohit and Raina [9].

Definition 1 (fractional $q$-integral operator). The fractional $q$ integral operator $I_{q, z}^{\delta}$ of a function $f(z)$ of order $\delta$ is defined by

$$
\begin{aligned}
I_{q, z}^{\delta} f(z) & \equiv D_{q, z}^{-\delta} f(z) \\
& =\frac{1}{\Gamma_{q}(\delta)} \int_{0}^{z}(z-t q)_{\delta-1} f(t) d_{q} t, \quad(\delta>0)
\end{aligned}
$$

where $f(z)$ is analytic in a simply connected region of the $z$ plane containing the origin and the $q$-binomial function $(z-$ $t q)_{\delta-1}$ is given by

$$
(z-t q)_{\delta-1}=z_{1}^{\delta-1} \Phi_{0}\left[q^{-\delta+1} ;-; q, \frac{t q^{\delta}}{z}\right] .
$$

The series ${ }_{1} \Phi_{0}[\delta ;-; q, z]$ is single valued when $|\arg (z)|<$ $\pi$ and $|z|<1$ (see for details [2], pp. 104-106); therefore, the function $(z-t q)_{\delta-1}$ in (15) is single valued when $\left|\arg \left(-t q^{\delta} / z\right)\right|<\pi,\left|t q^{\delta} / z\right|<1$, and $|\arg (z)|<\pi$.

Definition 2 (fractional $q$-derivative operator). The fractional $q$-derivative operator $D_{q, z}^{\delta}$ of a function $f(z)$ of order $\delta$ is defined by

$$
\begin{aligned}
& D_{q, z}^{\delta} f(z) \equiv D_{q, z} I_{q, z}^{1-\delta} f(z) \\
&=\frac{1}{\Gamma_{q}(1-\delta)} D_{q, z} \int_{0}^{z}(z-t q)_{-\delta} f(t) d_{q} t, \\
& \quad(0 \leq \delta<1),
\end{aligned}
$$

where $f(z)$ is suitably constrained and the multiplicity of $(z-$ $t q)_{-\delta}$ is removed as in Definition 1.

Definition 3 (extended fractional $q$-derivative operator). Under the hypotheses of Definition 2, the fractional $q$ derivative for a function $f(z)$ of order $\delta$ is defined by

$$
D_{q, z}^{\delta} f(z)=D_{q, z}^{m} I_{q, z}^{m-\delta} f(z),
$$

where $m-1 \leq \delta<1, m \in \mathbb{N}_{0}=\mathbb{N} \cup\{0\}$, and $\mathbb{N}$ denotes the set of natural numbers.

Remark 4. It follows from Definition 2 that

$$
D_{q, z}^{\delta} z^{n}=\frac{\Gamma_{q}(n+1)}{\Gamma_{q}(n+1-\delta)} z^{n-\delta}, \quad(\delta \geq 0, n>-1) .
$$

Using $D_{q, z}^{\delta}$, we define a $q$-differintegral operator $\Omega_{q, p}^{\delta}$ : $\mathscr{A}_{p} \rightarrow \mathscr{A}_{p}$, as follows:

$$
\begin{gathered}
\Omega_{q, p}^{\delta} f(z)=\frac{\Gamma_{q}(p+1-\delta)}{\Gamma_{q}(p+1)} z^{\delta} D_{q, z}^{\delta} f(z), \\
(-\infty<\delta<p+1 ; 0<q<1 ; z \in \mathcal{U}),
\end{gathered}
$$

where $D_{q, z}^{\delta} f(z)$ in (20) represents, respectively, a fractional $q$ integral of $f(z)$ of order $\delta$ when $-\infty<\delta<0$ and a fractional $q$-derivative of $f(z)$ of order $\delta$ when $0 \leq \delta<p+1$. It is easy to see from $(20)$ that

$$
\begin{array}{r}
\Omega_{q, p}^{\delta} f(z)=z^{p}+\sum_{n=p+1}^{\infty} \frac{\Gamma_{q}(p+1-\delta) \Gamma_{q}(n+1)}{\Gamma_{q}(p+1) \Gamma_{q}(n+1-\delta)} a_{n} z^{n}, \\
q^{j} z D_{q}^{j+1}\left(\Omega_{q, p}^{\delta} f(z)\right)=q^{\delta}[p-\delta]_{q} D_{q}^{j}\left(\Omega_{q, p}^{\delta+1} f(z)\right) \\
+q^{j}[\delta-j]_{q} D_{q}^{j}\left(\Omega_{q, p}^{\delta} f(z)\right), \\
(0 \leq j \leq p ;-\infty<\delta<p ; z \in \mathcal{U}) .
\end{array}
$$


Definition 5. A function $f(z) \in \mathscr{A}_{p}$ is said to be in the class $S_{q, p}^{\delta, j}(b)$ of $p$-valent functions of complex order $b \neq 0$ in $\mathcal{U}$ if and only if

$$
\begin{gathered}
\operatorname{Re}\left\{1+\frac{1}{b}\left(\frac{z D_{q}^{j+1}\left(\Omega_{q, p}^{\delta} f(z)\right)}{D_{q}^{j}\left(\Omega_{q, p}^{\delta} f(z)\right)}-[p-j]_{q}\right)\right\}>0, \\
\left(z \in \mathcal{U} ; p \in \mathbb{N} ; j \in \mathbb{N}_{0} ; b \in \mathbb{C}-\{0\} ;\right. \\
\left.\left|2 b q^{j-\delta}-[p-\delta]_{q}\right| \leq[p-\delta]_{q}\right) .
\end{gathered}
$$

It can be seen that, by specializing the parameters, the class $S_{q, p}^{\delta, j}(b)$ reduces to many known subclasses of analytic functions. For instance, if $q \rightarrow 1$ then

(1) $\mathcal{S}_{1,1}^{0,0}(b)=\delta(b)$, the class of starlike functions of complex order $b$ (see [14]),

(2) $\mathcal{S}_{1,1}^{1,0}(b)=\mathscr{C}(b)$, the class of convex functions of complex order $b$ (see [15]),

(3) $\mathcal{\delta}_{1,1}^{0,0}\left(\cos \alpha e^{-i \alpha}\right)=\mathcal{S}^{\alpha},(|\alpha|<\pi / 2)$, the class of $\alpha$ spiral-like functions (see [16]),

(4) $\mathcal{S}_{1,1}^{0,0}(1-\alpha)=\mathcal{S}^{*}(\alpha),(0 \leq \alpha<1)$, the class of starlike functions of order $\alpha$.

\section{Majorization Problem for the Class $S_{q, p}^{\delta, j}(b)$}

We start by proving the following $q$-analogue of the result given by Nehari in [17].

Lemma 6. If $f(z)$ is analytic and bounded in $\mathcal{U}$, then

$$
\left|D_{q}(f(z))\right| \leq \frac{1-|f(z)|^{2}}{1-|z|^{2}}, \quad(z \in \mathcal{U}) \text {. }
$$

Proof. If $f(z)$ is bounded in $\mathcal{U}$, then

$$
g(z)=\frac{f(z)-f(z q)}{1-\overline{f(z q)} f(z)}, \quad(0<q<1)
$$

is also bounded in $\mathcal{U}$. Clearly $g(z)$ vanishes when $q=1$. Therefore, the function

$$
\begin{aligned}
h(z) & =\frac{g(z)}{((z-z q) /(1-z \bar{z} q))} \\
& =\left(\frac{f(z)-f(z q)}{z-z q}\right)\left(\frac{1-z \bar{z} q}{1-\overline{f(z q)} f(z)}\right)
\end{aligned}
$$

is regular when $q=1$ and also at all other points of $|z|<1$. Furthermore, $h(z)$ is bounded in $|z|<1$. In fact, $\lim _{|z| \rightarrow 1}|g(z)| \leq 1$ and $|(z-z q) /(1-z \bar{z} q)|=1$ for $|z|=1$; hence by maximum principle, $|h(z)| \leq 1$ throughout $|z|<1$. Then from (26) we have

$$
\begin{aligned}
& \left|D_{q}(f(z))\right|\left(\frac{1-|z|^{2}}{1-|f(z)|^{2}}\right) \leq 1 \\
& \text { which implies }\left|D_{q}(f(z))\right| \leq \frac{1-|f(z)|^{2}}{1-|z|^{2}} .
\end{aligned}
$$

Theorem 7. Let the function $f(z)$ be in the class $\mathscr{A}_{p}$ and suppose that $g(z) \in S_{q, p}^{\delta, j}(b)$. If $D_{q}^{j}\left(\Omega_{q, p}^{\delta} f(z)\right)$ is majorized by $D_{q}^{j}\left(\Omega_{q, p}^{\delta} g(z)\right)$ in $\mathcal{U}$ for $j \in \mathbb{N}_{0}$, then

$$
\left|D_{q}^{j}\left(\Omega_{q, p}^{\delta+1} f(z)\right)\right| \leq\left|D_{q}^{j}\left(\Omega_{q, p}^{\delta+1} g(z)\right)\right| \quad \text { for }|z| \leq r_{1},
$$

where

$$
\begin{aligned}
r_{1} & =r_{q}(p, j, \delta ; b) \\
& :=\frac{k-\sqrt{k^{2}-4[p-\delta]_{q}\left|2 b q^{j-\delta}-[p-\delta]_{q}\right|}}{2\left|2 b q^{j-\delta}-[p-\delta]_{q}\right|} \\
& \left(k:=2 q^{j-\delta}+[p-\delta]_{q}+\left|2 b q^{j-\delta}-[p-\delta]_{q}\right| ;\right. \\
& p \in \mathbb{N} ; b \in \mathbb{C}-\{0\} ; 0<q<1 ; \delta \geq 0) .
\end{aligned}
$$

Proof. Let

$$
h(z)=1+\frac{1}{b}\left(\frac{z D_{q}^{j+1}\left(\Omega_{q, p}^{\delta} g(z)\right)}{D_{q}^{j}\left(\Omega_{q, p}^{\delta} g(z)\right)}-[p-j]_{q}\right)
$$

$\left(z \in \mathcal{U} ; b \in \mathbb{C}-\{0\} ; p \in \mathbb{N} ; j \in \mathbb{N}_{0}\right.$ and $\left.j<p\right)$.

Since $g(z) \in S_{q, p}^{\delta, j}(b)$, we have $\operatorname{Re}(h(z))>0(z \in \mathcal{U})$ and

$$
h(z)=\frac{1+w(z)}{1-w(z)}, \quad(w \in \mathscr{P}),
$$

where $\mathscr{P}$ denotes the well known class of bounded analytic functions in $\mathscr{U}$, which satisfy the conditions (cf. [18])

$$
w(0)=0, \quad|w(z)| \leq|z| \quad(z \in \mathcal{U}) .
$$

It follows from (30) and (31) that

$$
\frac{z D_{q}^{j+1}\left(\Omega_{q, p}^{\delta} g(z)\right)}{D_{q}^{j}\left(\Omega_{q, p}^{\delta} g(z)\right)}=\frac{[p-j]_{q}+\left(2 b-[p-j]_{q}\right) w(z)}{1-w(z)}
$$

In view of the identity (22), we have the following inequality from (33) by making some simple calculations:

$$
\begin{aligned}
\left|D_{q}^{j}\left(\Omega_{q, p}^{\delta} g(z)\right)\right| \\
\leq \frac{[p-\delta]_{q}(1+|z|)}{[p-\delta]_{q}-\left|2 b q^{j-\delta}-[p-\delta]_{q}\right||z|} \\
\quad \times\left|D_{q}^{j}\left(\Omega_{q, p}^{\delta+1} g(z)\right)\right| .
\end{aligned}
$$

Since $D_{q}^{j}\left(\Omega_{q, p}^{\delta} f(z)\right)$ is majorized by $D_{q}^{j}\left(\Omega_{q, p}^{\delta} g(z)\right)$ in $\mathcal{U}$, there exists an analytic function $\varphi(z)$ such that

$$
D_{q}^{j}\left(\Omega_{q, p}^{\delta} f(z)\right)=\varphi(z) D_{q}^{j}\left(\Omega_{q, p}^{\delta} g(z)\right),
$$


and $|\varphi(z)| \leq 1 \quad(z \in \mathcal{U})$. Applying $q$-differentiation with respect to $z$ and multiplying by $z$, we have from (35)

$$
\begin{aligned}
z D_{q}^{j+1}\left(\Omega_{q, p}^{\delta} f(z)\right)= & z D_{q}(\varphi(z)) D_{q}^{j}\left(\Omega_{q, p}^{\delta} g(z)\right) \\
& +z \varphi(z) D_{q}^{j+1}\left(\Omega_{q, p}^{\delta} g(z)\right) .
\end{aligned}
$$

Using (22), in the above equation, we get

$$
\begin{aligned}
D_{q}^{j}\left(\Omega_{q, p}^{\delta+1} f(z)\right)= & \frac{z D_{q}(\varphi(z))}{q^{\delta-j}[p-\delta]_{q}} D_{q}^{j}\left(\Omega_{q, p}^{\delta} g(z)\right) \\
& +\varphi(z) D_{q}^{j}\left(\Omega_{q, p}^{\delta+1} g(z)\right) .
\end{aligned}
$$

Noting that $\varphi(z)$ is bounded in $\mathscr{U}$ and using Lemma 6 we obtain

$$
\left|D_{q}(\varphi(z))\right| \leq \frac{1-|\varphi(z)|^{2}}{1-|z|^{2}}, \quad(z \in \mathcal{U}) .
$$

Appling (34) and (38) in (37) we get

$$
\begin{aligned}
\mid & D_{q}^{j}\left(\Omega_{q, p}^{\delta+1} f(z)\right) \mid \\
\leq & \left\{\varphi(z)+\frac{1-|\varphi(z)|^{2}}{1-|z|}\right. \\
& \left.\cdot \frac{q^{j-\delta}|z|}{[p-\delta]_{q}-\left|2 b q^{j-\delta}-[p-\delta]_{q}\right||z|}\right\} \\
= & \frac{-q^{j-\delta} r \rho^{2}+(1-r)\left([p-\delta]_{q}-\left|2 b q^{j-\delta}-[p-\delta]_{q}\right| r\right) \rho+q^{j-\delta} r}{(1-r)\left([p-\delta]_{q}-\left|2 b q^{j-\delta}-[p-\delta]_{q}\right| r\right)} \\
& \times\left|D_{q}^{j+1}\left(\Omega_{q, p}^{\delta+1} g(z)\right)\right| \quad(|z|=r,|\varphi(z)|=\rho, 0 \leq \rho \leq 1) \\
= & \frac{\Theta(\rho)}{(1-r)\left([p-\delta]_{q}-\left|2 b q^{j-\delta}-[p-\delta]_{q}\right| r\right)} \\
& \times\left|D_{q}^{j}\left(\Omega_{q, p}^{\delta+1} g(z)\right)\right| \quad(z \in \mathscr{U}),
\end{aligned}
$$

where the function $\Theta(\rho)$, defined by

$$
\begin{aligned}
\Theta(\rho):= & -q^{j-\delta} r \rho^{2}+(1-r) \\
& \times\left([p-\delta]_{q}-\left|2 b q^{j-\delta}-[p-\delta]_{q}\right| r\right) \rho+q^{j-\delta} r, \\
& (0 \leq \rho \leq 1),
\end{aligned}
$$

takes its maximum value at $\rho=1$ with $r=r_{q}(p, j, \delta ; b)$ given by (29). Furthermore, if $0 \leq \sigma \leq r_{q}(p, j, \delta ; b)$ where $r_{q}(p, j, \delta ; b)$ given by $(29)$, then the function

$$
\begin{aligned}
\Phi(\rho):= & -q^{j-\delta} \sigma \rho^{2}+(1-\sigma) \\
& \times\left([p-\delta]_{q}-\left|2 b q^{j-\delta}-[p-\delta]_{q}\right| \sigma\right) \rho+q^{j-\delta} \sigma
\end{aligned}
$$

increases in the interval $0 \leq \rho \leq 1$, so that $\Phi(\rho)$ does not exceed

$$
\begin{array}{r}
\Phi(1)=(1-\sigma)\left([p-\delta]_{q}-\left|2 b q^{j-\delta}-[p-\delta]_{q}\right| \sigma\right), \\
\left(0 \leq \sigma \leq r_{q}(p, j, \delta ; b)\right) .
\end{array}
$$

Therefore, from this fact, (39) gives inequality (28).

Letting $q \rightarrow 1, p=1, \delta=0$, and $j=0$ in Theorem 7 , we have the following.

Corollary 8 (see [19]). Let the function $f(z) \in \mathscr{A}$ be analytic and univalent in the open unit disk $\mathcal{U}$ and suppose that $g(z) \epsilon$ $\delta(b)$, the class of starlike functions of complex order $b$. If $f(z)$ is majorized by $g(z)$ in $\mathcal{U}$, then

$$
\left|f^{\prime}(z)\right| \leq\left|g^{\prime}(z)\right| \quad \text { for }|z| \leq r_{2},
$$

where

$$
r_{2}:=\frac{3+|2 b-1|-\sqrt{9+2|2 b-1|+|2 b-1|^{2}}}{2|2 b-1|} .
$$

For $b=\cos \alpha e^{-i \alpha}$, Corollary 8 reduces to the following result.

Corollary 9. Let the function $f(z) \in \mathscr{A}$ be analytic and univalent in the open unit disk $\mathcal{U}$ and suppose that $g(z) \in$ $\mathcal{S}^{\alpha}(|\alpha|<\pi / 2)$, the class of $\alpha$-spiral-like functions. If $f(z)$ is majorized by $g(z)$ in $\mathcal{U}$, then $\left|f^{\prime}(z)\right| \leq\left|g^{\prime}(z)\right|$ for $|z| \leq 2-\sqrt{3}$.

Further setting $b=1$, in Corollary 8 we get the following.

Corollary 10 (see [1]). Let the function $f(z) \in \mathscr{A}$ be analytic and univalent in the open unit disk $\mathscr{U}$ and suppose that $g(z)$ is starlike in $\mathcal{U}$. If $f(z)$ is majorized by $g(z)$ in $\mathcal{U}$, then

$$
\left|f^{\prime}(z)\right| \leq\left|g^{\prime}(z)\right| \quad \text { for }|z| \leq 2-\sqrt{3} \text {. }
$$

\section{Conflict of Interests}

The authors declare that there is no conflict of interests regarding the publication of this paper.

\section{References}

[1] T. H. MacGregor, "Majorization by univalent functions," Duke Mathematical Journal, vol. 34, pp. 95-102, 1967.

[2] G. Gasper and M. Rahman, Basic Hypergeometric Series, vol. 35 of Encyclopedia of Mathematics and Its Applications, Cambridge University Press, Cambridge, UK, 1990.

[3] H. Aldweby and M. Darus, "A subclass of harmonic univalent functions associated with $q$-analogue of Dziok-Srivastava operator," ISRN Mathematical Analysis, vol. 2013, Article ID 382312, 6 pages, 2013.

[4] H. Aldweby and M. Darus, "On harmonic meromorphic functions associated with basic hypergeometric functions," The Scientific World Journal, vol. 2013, Article ID 164287, 7 pages, 2013. 
[5] H. Aldweby and M. Darus, "Some subordination results on $q$-analogue of Ruscheweyh differential operator," Abstract and Applied Analysis, vol. 2014, Article ID 958563, 6 pages, 2014.

[6] G. Murugusundaramoorthy, C. Selvaraj, and O. S. Babu, "Subclasses of starlike functions associated with fractional $q$-calculus operators," Journal of Complex Analysis, vol. 2013, Article ID 572718, 8 pages, 2013.

[7] G. Murugusundaramoorthy and T. Janani, "Meromorphic parabolic starlike functions associated with $q$-hypergeometric series," ISRN Mathematical Analysis, vol. 2014, Article ID 923607, 9 pages, 2014.

[8] S. D. Purohit, "A new class of multivalently analytic functions associated with fractional $q$-calculus operators," Fractional Differential Calculus, vol. 2, no. 2, pp. 129-138, 2012.

[9] S. D. Purohit and R. K. Raina, "Certain subclasses of analytic functions associated with fractional q-calculus operators," Mathematica Scandinavica, vol. 109, no. 1, pp. 55-70, 2011.

[10] S. D. Purohit and R. K. Raina, "Fractional $q$-calculus and certain subclass of univalent analytic functions," Mathematica, vol. 55, no. 1, pp. 62-74, 2013.

[11] S. D. Purohit and R. K. Raina, "Some classes of analytic and multivalent functions associated with q-derivative operators," Acta Universitatis Sapientiae, Mathematica, vol. 6, no. 1, 2014.

[12] S. D. Purohit and R. K. Raina, "On a subclass of $p$-valent analytic functions involving fractional $q$-calculus operators," Kuwait Journal of Science, vol. 41, no. 3, 2014.

[13] K. A. Selvakumaran, S. D. Purohit, A. Secer, and M. Bayram, "Convexity of certain $q$-integral operators of $p$-valent functions," Abstract and Applied Analysis, vol. 2014, Article ID 925902, 7 pages, 2014.

[14] M. A. Nasr and M. K. Aouf, "Starlike function of complex order," The Journal of Natural Sciences and Mathematics, vol. 25, no. 1, pp. 1-12, 1985.

[15] P. Wiatrowski, "The coefficients of a certain family of holomorphic functions," Zeszyty Naukowe Uniwersytetu Lodzkiego, Nauki Matematyczno Przyrodnicze II, Zeszyt, no. 39, pp. 75-85, 1971.

[16] R. J. Libera, “Univalent $\alpha$-spiral functions," Canadian Journal of Mathematics, vol. 19, pp. 449-456, 1967.

[17] Z. Nehari, Conformal Mapping, McGraw-Hill, New York, NY, USA, 1952.

[18] A. W. Goodman, Univalent Functions, Mariner, Tampa, Fla, USA, 1983.

[19] O. Altintaş, Ö. Özkan, and H. M. Srivastava, "Majorization by starlike functions of complex order," Complex Variables: Theory and Application, vol. 46, no. 3, pp. 207-218, 2001. 


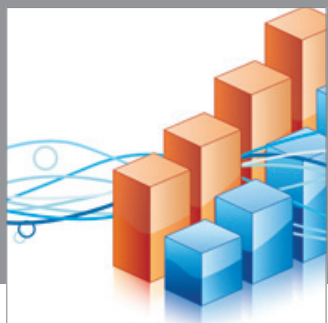

Advances in

Operations Research

mansans

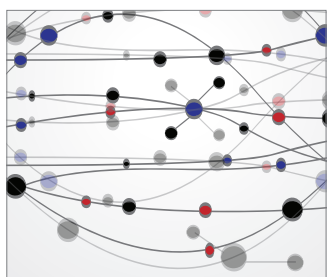

The Scientific World Journal
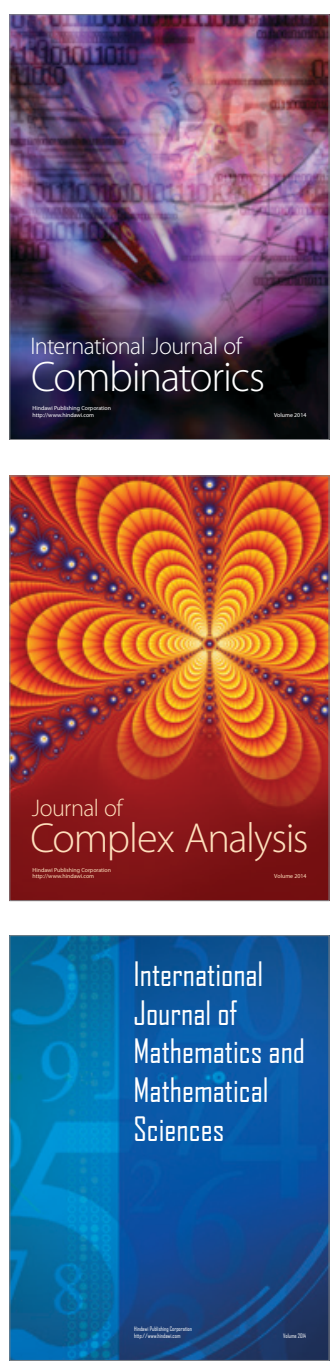
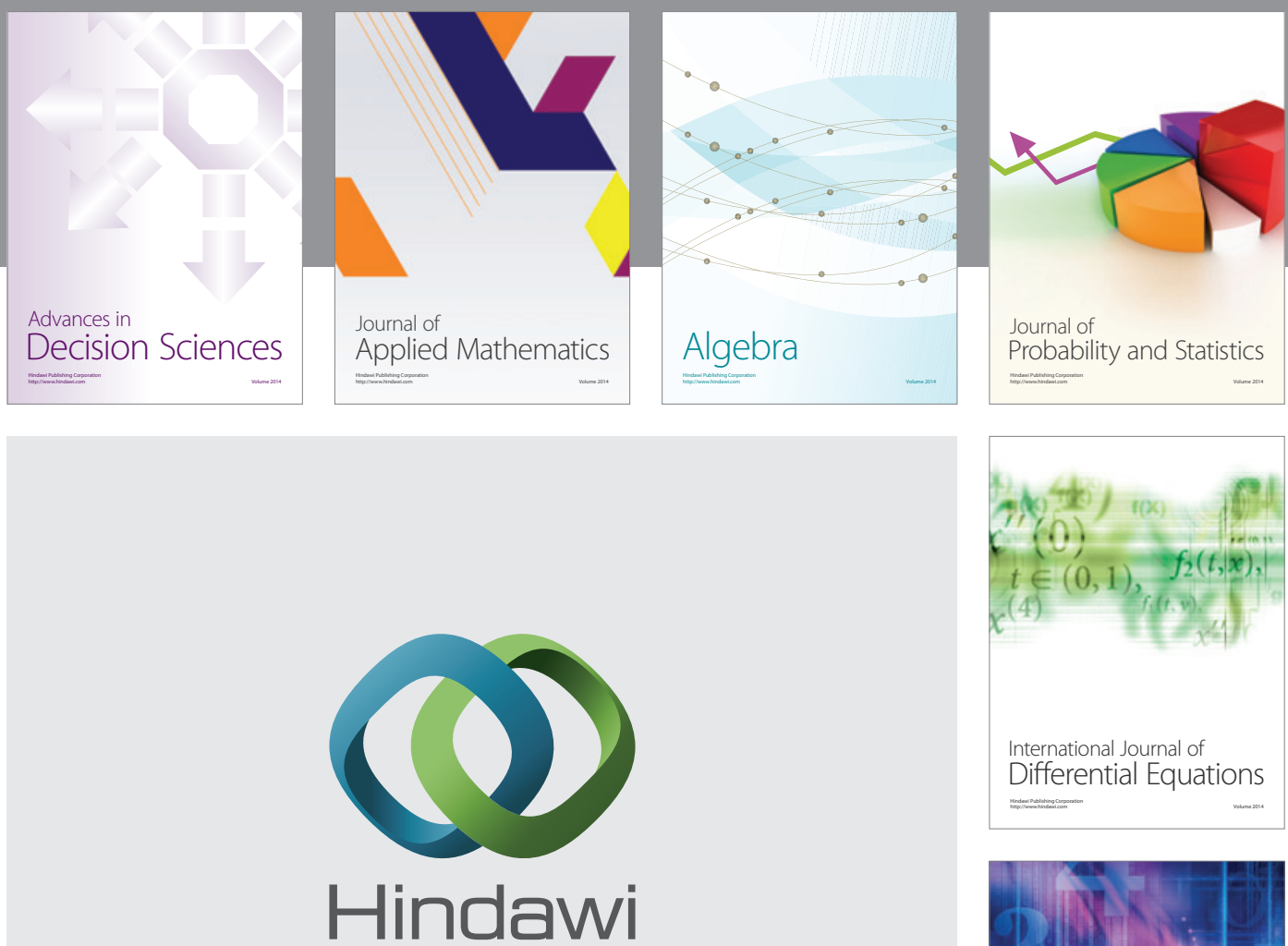

Submit your manuscripts at http://www.hindawi.com
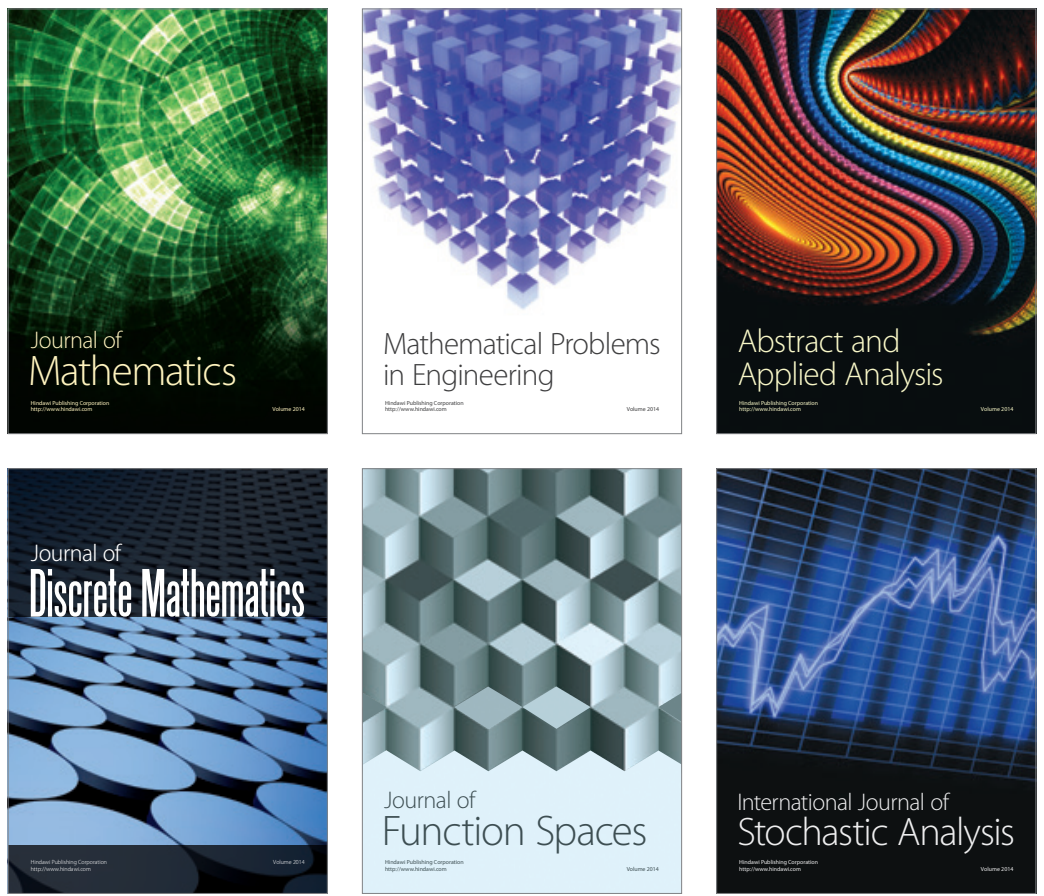

Journal of

Function Spaces

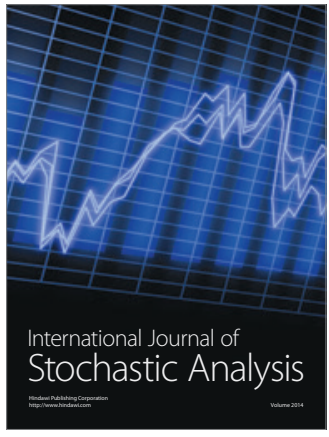

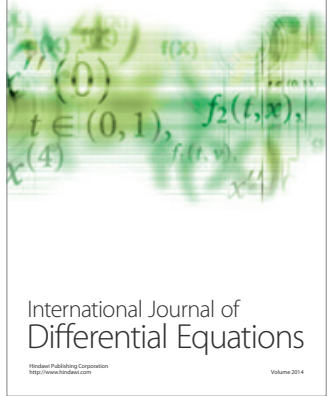
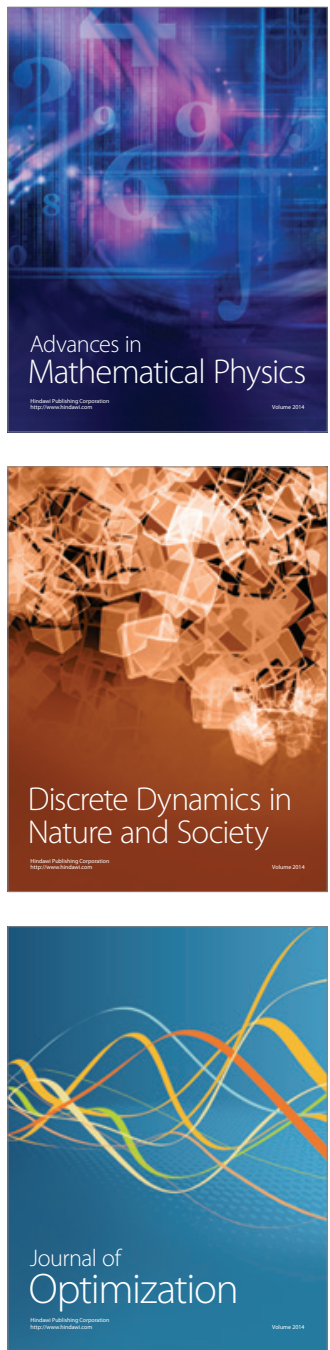